\title{
Retrograde and anterograde amnesia for spatial discrimination in rats: Role of hippocampus, entorhinal cortex, and parietal cortex
}

\author{
YOON H. CHO, RAYMOND P. KESNER, and SEAN BRODALE \\ University of Utah, Salt Lake City, Utah
}

\begin{abstract}
The present study was conducted to examine whether lesions of hippocampus (HIP), entorhinal cortex (EC), or parietal cortex (PC) in rats produce retrograde amnesia for spatial two-choice discriminations in an eight-arm radial maze. Rats were trained successively on two single-pair discriminations, and lesions were performed approximately $24 \mathrm{~h}$ after the rat had acquired the second discrimination. After recovery from surgery, the animals were tested for retention of the two preoperatively acquired pairs at least for 2 days, and training continued until the animals reattained criterion performance for each of the pairs. Results indicated that when the two discrimination pairs were tested concurrently (Experiment 1), the HIP- and EC-lesioned rats were impaired in retention of the two presurgically acquired discriminations, whereas the $\mathrm{PC}$ lesioned rats displayed retention levels similar to the sham lesioned controls (SH). However, the EC- and PC-lesioned groups of animals were normal in reacquiring postoperatively the two pairs concurrently and in acquiring a new one-pair discrimination, whereas the HIP-lesioned rats displayed retarded relearning. Furthermore, under conditions in which the two pairs were tested successively, one at a daily session (Experiment 2), the two lesioned groups (HIP and EC) were still impaired in retention as well as in relearning of the discriminations in comparison with the control group. The HIP-lesioned rats, however, exhibited a significant improvement of performance in comparison with that of the HIP-lesioned rats in Experiment 1. The EC-lesioned rats displayed higher retention scores for the first (remote) than for the second (recent) pair acquired prior to surgery (temporal gradient). Furthermore, both the HIP- and the EC-lesioned rats were slower in acquisition of a new two-pair concurrent discrimination. These results were discussed in the context of current theories of learning and memory.
\end{abstract}

Clinical literature has indicated that human patients who have suffered head injuries tend to have difficulty recalling events that occurred shortly before injury, even though older memories might be intact. This phenomenon, called retrograde amnesia (RA), constitutes an intriguing aspect of human memory pathology. Attempts to produce an analogous deficit in infrahuman species have been numerous. Most such studies have employed amnesic treatments, such as electroconvulsive shock, cortical spreading depression, hypothermia, or protein synthesis inhibition. The memory tasks used have very often been limited to passive avoidance or classical conditioning (Bures \& Buresova, 1963; Chorover \& Schiller, 1965; Flexner, Flexner \& Stellar, 1963; Gold \& Sternberg, 1978; McGaugh, 1966). However, animal experiments can offer considerable advantages over human studies, since the exact date or duration of presurgical events can be controlled. Only recently have lesions been made of the hippocampus (HIP) or related structures in order to study RA, resulting in varied patterns of behavioral results in nonhuman primates and in rodents (Gaffan, 1993;

Correspondence should be addressed to $\mathrm{Y}$. H. Cho, who is now at the Center for Behavioral Neuroscience, State University of New York, Stony Brook, NY 11794-2575.
Kim \& Fanselow, 1992; Salmon, Zola-Morgan, \& Squire, 1987; Staubli, Fraser, Kessler, \& Lynch, 1986; Winocur, 1990; Zola-Morgan \& Squire, 1990).

In one study, mice were trained on a successive twochoice spatial discrimination task $0.5,2.5,4.5$, and 7 weeks before bilateral ibotenate lesions of entorhinal cortex (EC). Behavioral results indicated that damage to the EC produced a temporally graded RA for spatial discrimination memory in mice (Cho, Beracochea, \& Jaffard, 1993). That is, the EC lesions resulted in a marked impairment in retention of spatial discriminations acquired immediately and up to 4 weeks prior to surgery, but had no effect on discriminations learned beyond 4 weeks before surgery. Together with earlier reports (Kim \& Fanselow, 1992; Winocur, 1990; Zola-Morgan \& Squire, 1990), these data suggest that some temporal structures are important for memory after learning, and that beyond this time interval, the involvement of these regions is not critical for normal retrieval of stored memory representations. Furthermore, it can be shown that the time scale necessary to demonstrate an RA effect varies with a number of variables, such as learning material, level of training, species used, and number of learning materials (Olton \& Shapiro, 1992; Squire, 1992).

Another advantage of experimental animal research on RA is that it offers a powerful insight into the exact 
nature of memory processing and storage associated with parametric manipulation of learning materials. In the present study, we thus aimed at determining the generality of RA for spatial discrimination by varying some of the parameters and maintaining others. So, for example, rats were used in the present study instead of mice. Furthermore, a smaller number of discrimination problems was used without a lengthy time interval between the problems, so that learning-to-surgery delays were shortened. Finally, HIP- as well as parietal-cortex(PC-) lesioned groups were included in the study, for the following reasons. Neuroanatomical as well as electrophysiological data have indicated that hippocampalcortical interplay should be considered critical for memory processing as well as for long-term memory storage. Posterior association cortex, however, has received little, if any, attention in studies of RA, even though it has often been considered as a permanent memory storage site (Eichenbaum, Otto, \& Cohen, 1994; Squire, Cohen, \& Nadel, 1984). Within the posterior association cortex, PC in rats has been reported to be important for organizing spatial behavior (Kesner \& DiMattia, 1984) and for containing the topological memory traces for their recall (DiMattia \& Kesner, 1988a; Kesner, Farnworth, \& DiMattia, 1989; Kolb \& Walkey, 1987). The present research was thus designed to determine the contribution of the PC as well as the HIP and $\mathrm{EC}$ in rats to retention memory of preoperatively acquired spatial memory.

Another objective of the present research was to study separately anterograde and retrograde amnesia. One approach to accomplishing this goal is to compare simultaneous and concurrent discrimination tasks, which have been suggested as implicating differential memory demands as well as involvement of the HIP (Olton \& Shapiro, 1992).

\section{EXPERIMENT 1}

The first experiment was designed to determine whether lesions of the HIP, EC, and PC produce deficit in retention of two presurgically acquired one-pair discriminations in rats. Thus, rats were successively trained on two one-pair discriminations, and lesions of the HIP, $\mathrm{EC}$, or PC were performed approximately $24 \mathrm{~h}$ after the rats had acquired the second pair. Following recovery from surgery, the animals were tested for retention of the two presurgically acquired pairs by using trials in which the two pairs were tested concurrently within each of the testing sessions. The animals were further trained until they reattained presurgical criterion performance. The animals were subsequently trained on a new one-pair discrimination that had not previously been used. On the basis of previous reports indicating that lesions of the hippocampus alone or combined with the parahippocampal region produce temporally graded RA in rats (Kim \& Fanselow, 1992; Winocur, 1990) and monkeys (Zola-Morgan \& Squire, 1990), the prediction would be that lesions of both the HIP and the EC should produce temporally graded RA. In addition, lesions of the parietal cortex may not result in temporal gradient of retrograde amnesia because this structure is considered as the final repository of long-term memory.

\section{Method}

\section{Subjects}

Twenty-two Long-Evans male rats, approximately 4 months old at the beginning of the experiment, were used as subjects. The rats were individually housed in standard wire cages with food and water available at all times and maintained on a 13:11-h light:dark cycle. Behavioral testing was carried out during the light cycle. Before the start of training, the rats were progressively food deprived for a week so that their body weight was reduced to $85 \%$ of their free-feeding weight. This level of body weight was maintained during the whole experiment.

\section{Apparatus}

The apparatus used was an 8-arm radial maze constructed of wood and painted white. The maze consisted of a central hexagonal wooden platform, $41 \mathrm{~cm}$ in diameter, with eight arms, $61 \mathrm{~cm}$ long and $9 \mathrm{~cm}$ wide, projecting outward from the center platform. A food well ( $3 \mathrm{~cm}$ in diameter) was placed $0.5 \mathrm{~cm}$ from the end of each arm, and a clear Plexiglas door, $20 \mathrm{~cm}$ high, was placed between each arm and the central platform. Each door was attached by a string-and-pulley system to a control board adjacent to the maze room. The maze was placed in a $2.5 \times 1.75 \mathrm{~m}$ room and was elevated $1 \mathrm{~m}$ above the floor. The room was decorated with posters on the walls to provide the rats with a visual reference frame.

\section{Behavioral Procedure}

Prior to training, the food-deprived rats were first familiarized with the apparatus. The rats were given two sessions of free exploration, during which they were placed in the maze. The exploration session ended when they moved freely about the maze and consumed small pieces of food placed in the food well at the end of each arm. Half a piece of Froot Loop cereal was used as food reinforcement.

\section{Preoperative Training}

After familiarization with the apparatus, rats were trained in sequence on two-choice spatial discriminations involving two different pairs of arms of the maze. For each discrimination, one pair of adjacent arms was employed, one member of which was assigned as positive and thus associated with reinforcement throughout testing, whereas the other arm was assigned as negative. Each trial started with the opening of two doors, giving the rats access to the arms and providing them the opportunity to choose one of the two arms. Once animals had chosen an arm and had returned to the central platform, they were confined for an interval of $15 \mathrm{sec}$. After the interval, the same two doors were reopened for the next trial. A daily session consisted of 16 trials. The training continued until the rats attained criterion performance of 13 correct responses in 16 consecutive trials or 12 correct responses in two sets of 16 consecutive trials. On the day following completion of acquisition of the first pair, training on the second pair began, using the same procedure as that employed for the first one. The two pairs of arms were taken from spatially distinct areas of the maze (e.g., Arms 1 and 2, and 5 and 6), and the position (i.e., left or right) of reinforcement for each of the two pairs was counterbalanced (e.g., arm $1+$ vs. 2 -, and then $5-$ vs. $6+$ ) across subjects and groups. Preoperative acquisition performance of the two pairs was used for assignment of rats into one of four matched groups: sham-, HIP-, EC-, or PC-lesioned groups. Surgery was 
performed $24 \mathrm{~h}$ after rats had attained criterion performance on the second pair.

\section{Postoperative Retention Tests and Relearning}

After 1 week for recovery from surgery, the rats were assessed for retention of the two presurgically trained discriminations, and both pairs were presented concurrently within each session for 2 days, each of the two pairs being given eight times a day. Thus, retention memory scores (percent correct) were measured on the first 16 trials. The training continued until all rats reattained criterion performance for both of the discriminations concurrently (7 correct responses) in 8 successive trials (day) or 12 correct responses within 2 consecutive days ( 16 trials) for each of the pairs.

\section{Postoperative Learning of a New Pair}

On the day after reacquisition of both of the two pairs, animals were trained on a new single-pair discrimination involving a pair of arms that had not been used previously. Training continued until rats attained criterion performance.

\section{Surgery}

The rats that had completed preoperative training were assigned to the HIP- $(n=5)$, EC- $(n=6), \mathrm{PC}(n=5)$, or sham-lesioned ( $n$ $=6$ ) group on the basis of preoperative learning scores. For surgery, the animals were anesthetized with Nembutal $(50 \mathrm{mg} / \mathrm{kg}$, i.p.) and were given atropine sulfate $(0.2 \mathrm{mg} / \mathrm{kg}$, i.p.) as a prophylactic prior to surgery. The rats were then placed in a stereotaxic frame and were given a midline incision. Lesions of the HIP and EC were made electrolytically, whereas those of the $\mathrm{PC}$ were performed by aspiration.

For the HIP-lesioned group, a stainless steel electrode insulated with Epoxylite except for $0.5-0.75 \mathrm{~mm}$ at the tip was used in order to destroy the entire hippocampus. The coordinates used were $4.6 \mathrm{~mm}$ posterior to bregma, $1,2.2$, and $3.4 \mathrm{~mm}$ lateral to midline, and $2.8 \mathrm{~mm}$ below dura, and $5.6 \mathrm{~mm}$ posterior to bregma, $5.2 \mathrm{~mm}$ lateral to midline, and 5.6 and $8.1 \mathrm{~mm}$ from the top of the dura. Direct current ( $1.2 \mathrm{~mA}$ ) was applied for $10-20 \mathrm{sec}$ for each location. The coordinates used for lesions of the EC were $6.8 \mathrm{~mm}$ posterior to bregma, $4.3 \mathrm{~mm}$ and $5.4 \mathrm{~mm}$ lateral to midline, and $8.0 \mathrm{~mm}$ posterior to bregma and $5.0 \mathrm{~mm}$ lateral to midline. At each coordinate, the electrode was lowered to the bottom of the calvarium and then raised $1 \mathrm{~mm}$ for a $15-\mathrm{sec}$ lesion at each site. With respect to the PC group, the lesions were made bilaterally by means of aspiration and were intended to destroy parietal areas $5,7,39$, and 40 . For this, the skull was opened from $0.5 \mathrm{~mm}$ to $3.5 \mathrm{~mm}$ posterior to bregma and from $2 \mathrm{~mm}$ lateral to midline to the rhinal sulcus. All tissue up to the lateral ventricles was gently removed. Care was taken not to open the ventricles or to damage the underlying hippocampus.

\section{Histology}

At the end of the experiment, animals were given a lethal injection of sodium pentobarbital. The rats were then perfused with $10 \%$ buffered formalin in $0.1 \mathrm{M}$ phosphate buffer. The brain was then removed and embedded in a bovine serum albumin matrix. Then serial sections $(30 \mu \mathrm{m})$ were processed by using a freezing microtome throughout the extent of the lesion; one of every fifth section was mounted and stained with cresyl violet.

\section{Results}

\section{Histology}

All rats in the HIP-lesioned group exhibited acceptable lesions of the dorsal as well as ventral portion of the HIP. The exact extent of the damage was variable across animals. The lesions were extensive except for the medial portion of the HIP through the anteroposterior plane, and the most ventral and posterior part of the HIP was also spared from damage. As can be seen in Figure 1, some portion of the neocortical regions overlying the HIP was damaged, but the extent of cortical damage was not consistent across rats and was not systematically observed in the whole anteroposterior extent.

Examination of horizontal brain sections from the EClesioned rats revealed that all animals sustained acceptable bilateral damage to the EC (Figure 2). Across the subjects, there was damage bilaterally to the subicular complex, especially at the dorsal level, and bilaterally to the ventral subiculum at the most ventral portion of the EC. In 1 rat, some cells of the dentate gyrus were destroyed unilaterally at one dorsal-ventral level. In most of the animals, damage to the medial portion was more pronounced than that to the lateral portion of the EC, and dorsal perirhinal cortex was also damaged bilaterally in three of the animals.

The PC lesions were more or less complete across subjects (Figure 3). In the anterior-posterior plane, the lesions destroyed more massively the posterior than the anterior part of the PC. Microscopic examination of the rest of the brains revealed cell degeneration in the lateral posterior, posterior, and ventral posterior nuclei of the thala-

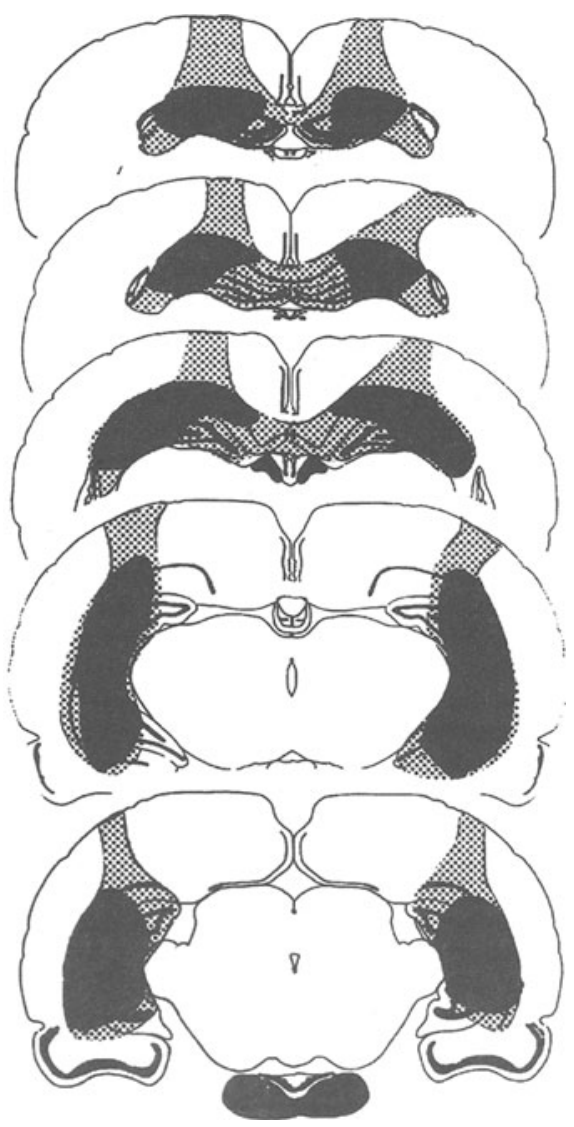

Figure 1. Reconstruction of the smallest (dark area) and the largest (dotted area) lesions of the hippocampus. 

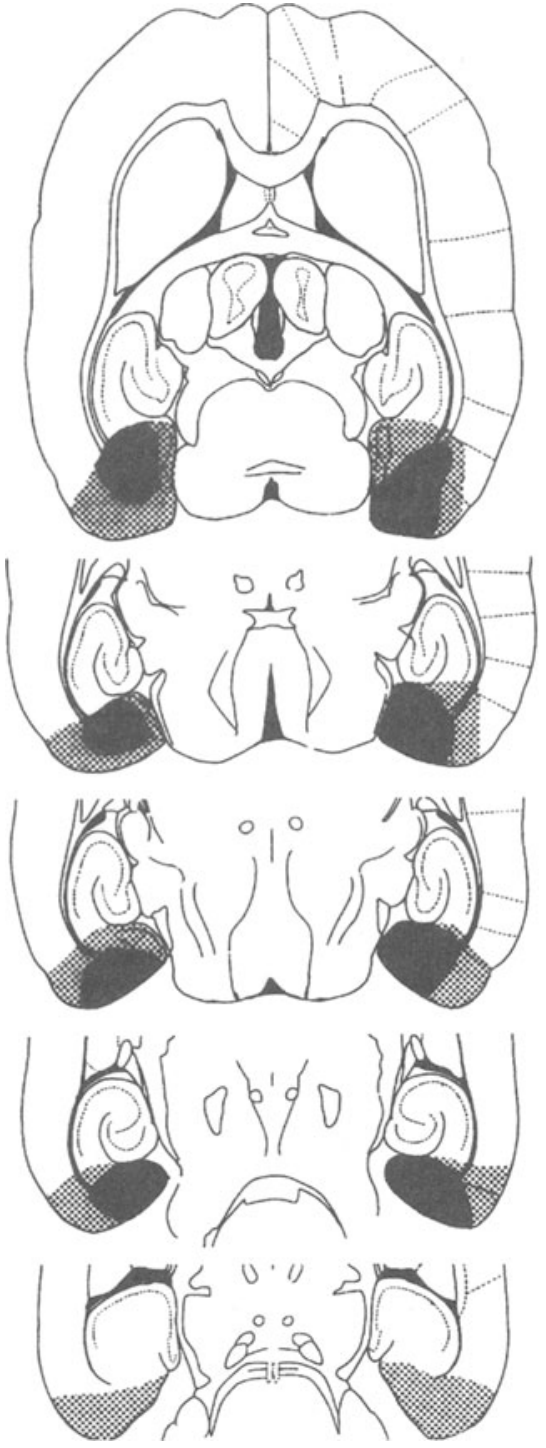

Figure 2. Reconstruction of the smallest (dark area) and the largest (dotted area) lesions of the entorhinal cortex.

mus, which indicates that the lesions destroyed the intended parietal areas.

\section{Behavior}

Prior to surgery, all rats had acquired the first pair in a mean of 3.59 sessions, and the second pair was learned faster ( 2.68 sessions) than the first one by all rats [trend analysis: $F(1,21)=8.71, p<.01]$. The total number of errors to attain criterion performance for both pairs did not differ ( 14.41 vs. 11.22 errors for the two pairs, respectively). The four groups were not significantly different from one another in acquiring both pairs prior to surgery $[F(3,18)<1$, n.s. $]$.

Postoperative retention performance is shown in Figure 4. A repeated measures analysis of variance (ANOVA) revealed a main effect of group $[F(3,18)=$
$8.23, p<.01]$, but no effect of pair $[F(1,18)<1$, n.s.] or interaction between the two variables $[F(3,18)<1$, n.s.]. Further post hoc Duncan multiple range tests indicated that the HIP- and EC-lesioned rats were impaired relative to the sham- or PC-lesioned animals $(p<.05)$. The sham- and PC-lesioned rats were not significantly different from each other, and the HIP-lesioned rats were not different from the EC-lesioned rats. A one-factor ANOVA performed for each of the pairs revealed a significant performance difference among the four groups for the second pair $[F(3,18)=3.20, p<.05]$, but not for the first one $(p>.05)$. Further analysis of the second pair revealed that the performance of both the HIP- and EClesioned rats was significantly different from that of the control group $(p<.05)$.

As can be seen in Figure 5, the four groups of subjects exhibited different numbers of errors for acquiring both of the pairs concurrently. A repeated measures

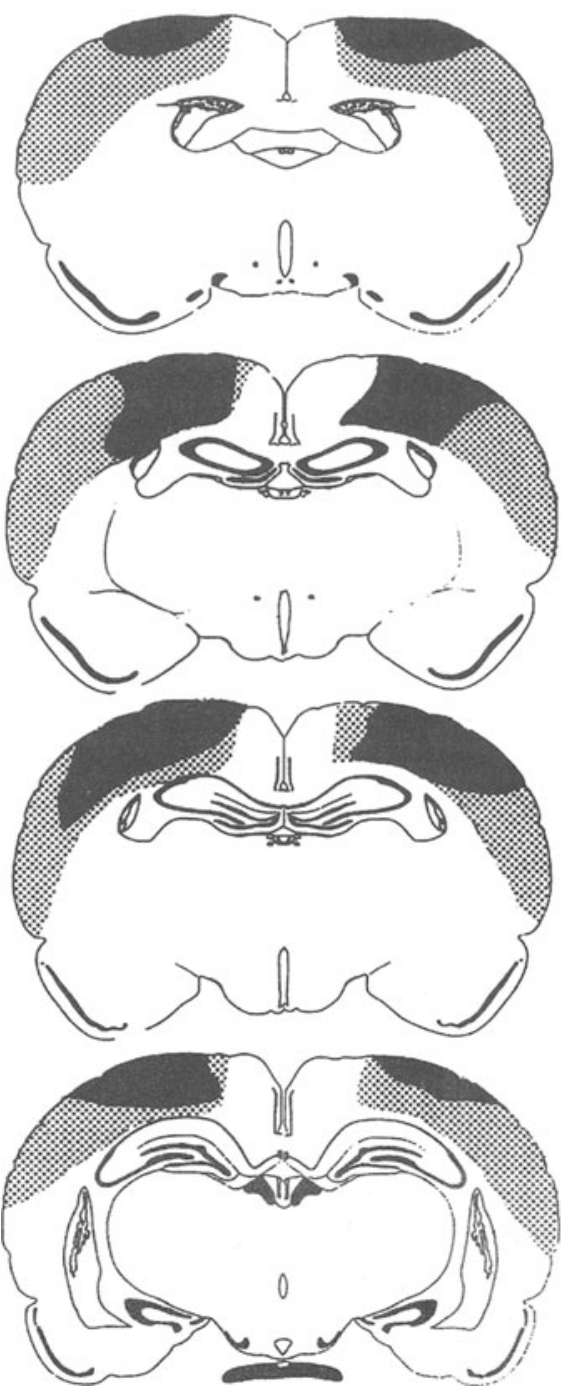

Figure 3. Reconstruction of the smallest (dark area) and the largest (dotted area) lesions of the parietal cortex. 


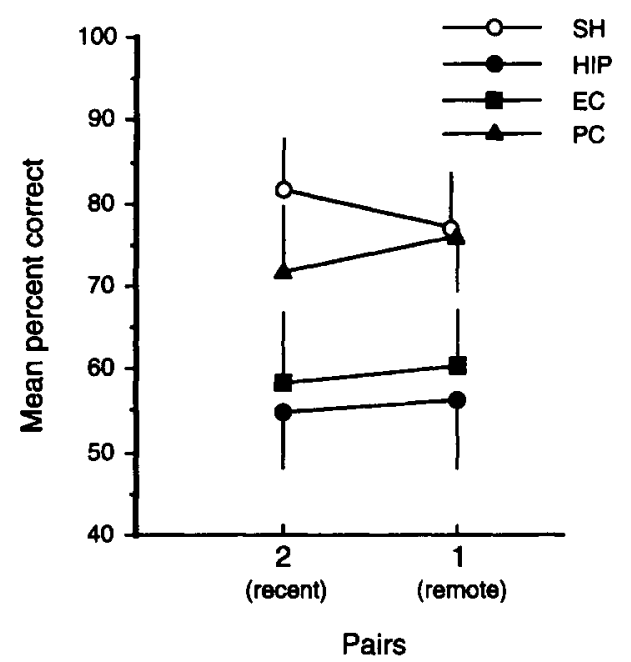

Figure 4. Postoperative retention performance (mean percent correct $+S E M)$ obtained on the first 16 trials for the two presurgically learned discriminations in the sham-lesioned (SH), hippocampallesioned (HIP), entorhinal-cortex-lesioned (EC), and parietalcortex-lesioned rats (PC). For retention memory testing, the preoperatively learned two pairs were assessed with concurrent paradigms (Experiment 1).

ANOVA revealed a significant main effect of group $[F(3,18)=3.85, p<.05]$. Further post hoc analysis revealed that the HIP group exhibited more errors than did the sham and PC groups $(p<.05)$, and the sham, $\mathrm{EC}$, and PC groups did not differ from one another $(p>.05)$. Finally, as shown in Figure 5, the four groups learned the new single-pair discrimination at a similar rate $[F(3.18)<1$, n.s. $)$.

\section{Discussion}

The present study showed that lesions of the EC produced a retention deficit of the one-pair discrimination learned 1 day before surgery, but the EC lesions resulted in a statistically nonsignificant performance difference for the pair acquired 3-5 days before surgery. The impairment does not appear to have been due to a relearning deficit (anterograde amnesia), since the EC-lesioned rats reacquired the two pairs as quickly as did controls. The data are consistent with previously reported observations in mice demonstrating that the $\mathrm{EC}$ is important for retention of spatial discriminations acquired immediately and up to 4 weeks prior to surgery (Cho et al., 1993).

The lesions of the HIP produced a retention as well as a relearning deficit, even though these rats were able to learn a new one-pair discrimination as readily as controls. There are several possible explanations for these results. First, it is possible that the acquisition-surgery time interval for both pairs (1-5 days) might have been too short to uncover differential consolidation times. Second, the deficit might have been due to an anterograde amnesic effect, since the rats were postoperatively tested under conditions where the two one-pair discriminations were presented concurrently, whereas they were trained successively (one at a time) during preoperative training. According to the relational model of HIP function (Eichenbaum, Otto, \& Cohen, 1992, 1994), changes in the testing conditions might represent a situation requiring the involvement of the HIP, because of the need to use flexibly previously stored memory representations. It has also been proposed that the HIP is critical for selecting temporal-spatial information, which can be accomplished by reducing interference among competing events (Olton \& Shapiro, 1992). Thus rats with HIP lesions would exhibit enhanced sensitivity to interference by the concurrent retention testing procedure. It is also possible that this anterograde amnesic effect might be due to enhanced temporal interference.

The PC lesions did not produce a notable deficit in retention of spatial discrimination, even though other studies have reported significant retention deficits of presurgically acquired Morris water maze or cheese board tasks

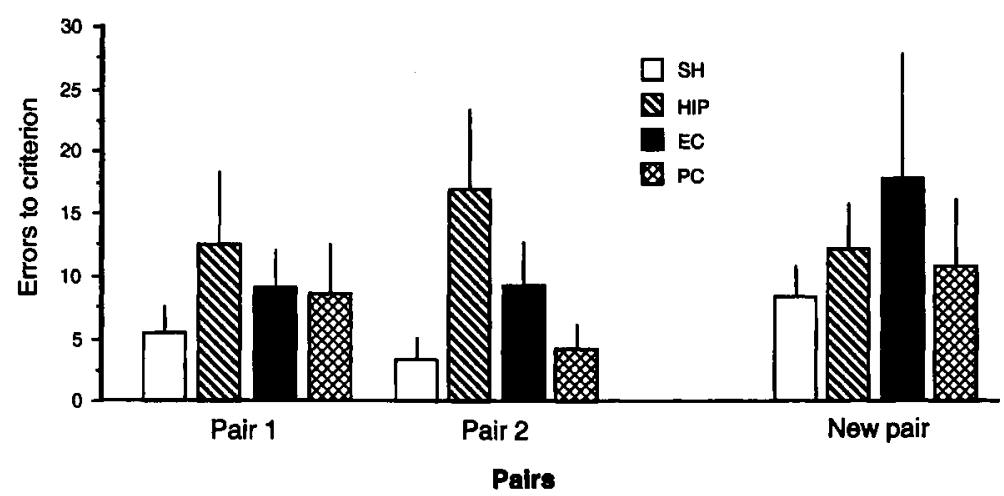

Figure 5. Mean errors ( $+S E M)$ required to reattain the two presurgically learned discriminations (Pair 1 and Pair 2), and mean errors needed to learn a new one-pair discrimination that had not been acquired presurgically in the sham-lesioned (SH), hippocampal-lesioned (HIP), entorhinal-cortex-lesioned (EC), and parietalcortex-lesioned (PC) rats. 
(DiMattia \& Kesner, 1988b; Kesner, Farnsworth, \& Kametani, 1991). However, impairment following the PC lesions has been less apparent (even in a working memory task) in radial maze tasks (DiMattia \& Kesner, 1988a; Juncker, Kametani, Bresnahan, \& Ingram, 1990) than in the previous Morris water maze tasks. These data, together with the present results, suggest the possibility that differences in spatial memory load between different mazes could influence the degree of the participation of the PC in spatial memory storage and retrieval.

\section{EXPERIMENT 2}

This experiment was conducted to determine whether the selection of a concurrent discrimination testing procedure and/or a change in testing procedure would result in anterograde amnesia and thus contribute to the retention deficit observed in the HIP- and EC-lesioned rats in Experiment 1. Thus, in this experiment the rats were tested for retention via a successive discrimination procedure. To further examine the possibility of deficit in relearning of the previously learned pairs (anterograde amnesia), the rats were given a new two-pair concurrent discrimination in which two pairs of stimuli were presented concurrently within sessions. A potential deficit in learning relative to controls would support the idea that massive retrograde amnesia without a temporal gradient for the EC- and HIP-lesioned rats in Experiment 1 might have been due to an anterograde amnesia. The PC group was not included in the present study, because their performance in the previous experiment was not different from that of controls despite the use of a concurrent memory testing procedure.

\section{Method}

\section{Subjects and Apparatus}

Fifteen adult male Long-Evans rats were used for this study, and the apparatus was the same as that used in Experiment 1.

\section{Procedure}

Prior to surgery, animals were trained on two one-pair discriminations in succession. The preoperative procedure employed was identical to that used in Experiment 1. Subjects were then assigned to three matched groups according to their presurgical performance and were given sham $(n=4)$, or $\operatorname{HIP}(n=5)$, or $\operatorname{EC~}(n=$ 6) lesions.

After a 1-week period of recovery, the animals were tested on retention memory of the two presurgically learned discriminations via the one-pair/session procedure for 2 consecutive days. The order of presentation of the two discriminations was counterbalanced across rats and groups; one half of the rats in each group were given Pair 1 on the first day and Pair 2 on the next day, whereas the other half of the rats in each group were tested in the reverse order. Retention memory scores were based on the number of correct responses over the first 16 postoperative trials for each of the problems. The rats were then trained on reacquisition of both pairs via the same procedure as that employed during the retention testing sessions. At the end of training, the rats were given acquisition of a new two-pair concurrent discrimination until they attained criterion performance of either 7 correct responses in 8 suc- cessive trials or 12 correct responses in 16 successive trials for both problems simultaneously.

\section{Surgery and Histology}

Surgical and histological procedures were the same as those in Experiment 1 .

\section{Results}

\section{Histology}

Careful examination of coronal sections from the HIPlesioned rats revealed acceptable lesions invading the dorsal as well as ventral portions of the HIP. As can be seen in Figure 1, the lesions included the whole extent of the HIP through the anterior-posterior as well as midlinelateral plane, except for the most medial and ventral portion of the HIP (dentate gyrus and CAl of the hippocampus). A variable degree of overlying cortical cells were also damaged, but the extent of cortical damage was not consistent across rats and was not systematically observed in the whole anterior-posterior extent.

Examination of horizontal brain sections from the EClesioned rats revealed that all subjects sustained near total damage to the EC, and the extent and placement of the lesions was similar to that observed in the EC-lesioned rats used in Experiment 1 (Figure 2). The lesions included the medial entorhinal cortex as well as the subicular complex in all 6 lesioned rats, whereas damage to the lateral entorhinal cortex, especially at the very ventral level, was partial in 3 rats. In addition, there was damage to perirhinal as well as temporal cortex (TE2) at the dorsal entorhinal cortex level in 3 animals, and some ventral subiculum cells as well as granule cells of dentate gyrus were also destroyed in some of the animals.

\section{Behavior}

Preoperatively, all animals acquired the second pair more quickly than the first pair [session to criterion, 3.80 vs. 2.13 sessions, respectively; trend analysis, $F(1,14)=$ $9.21, p<.01$; errors to criterion, 18.20 vs. 5.67 errors; trend analysis, $F(1,14)=10.46, p<.01]$. Finally, the three groups did not differ in acquiring both pairs preoperatively $[F(2,12)<1$, n.s. for both sessions and errors to criterion].

Postoperative retention performance, shown in Figure 6, indicated that the EC- and HIP-lesioned rats were markedly impaired relative to controls. A repeated measures ANOVA effectively revealed a main effect of group $[F(2,12)=10.16, p<.01]$, but there was no effect of pair $[F(1,12)<1$, n.s.] or interaction between the two variables $[F(2,12)<1$, n.s.]. (See Figure 6.) Further Duncan multiple range tests revealed that both lesioned groups were different from the sham group $(p<.05)$. A one-factor ANOVA performed on each of the pairs indicated a significant difference between the groups for the second pair $[F(2,12)=3.43, p<.05]$ but not for the first one $(p>.05)$. Further analysis for the second pair indicated a significant difference between the sham and EC 
groups $(p<.05)$. Within-group comparison analysis for performance on both of the pairs revealed that the EClesioned rats performed better for the first (remote) than for the second (recent) pair $(p<.05)$.

When the three groups were retrained on the two pairs, they exhibited different numbers of errors to reattain criterion performance $[F(2,12)=5.49, p<.05]$, but no significant difference between the pairs was observed. Further post hoc analysis indicated that both the HIP and EC groups made more errors than did the sham group $(p<$ .05 ), and that there was no significant difference between the two lesioned groups $(p>.05)$ (Figure 7).

Performance of a new two-pair concurrent discrimination is summarized in Figure 8. An ANOVA performed on the total number of errors to attain criterion performance for both of the pairs indicated a significant effect of group $[F(2,12)=1.98, p<.05]$. Further analysis indicated that the two lesioned groups exhibited more errors than did the sham groups $(p<.05)$.

An additional analysis, which differed mainly in the retention testing procedure (Figure 4 vs. 6), was performed in order to compare retention memory performance obtained in the two experiments. A repeated measures ANOVA with testing condition (Experiment 1 vs. 2 ) as the between-group factor and pair as the withingroup factor revealed that the HIP-lesioned rats exhibited better retention performance in the successive single-pair testing condition (Experiment 2, Figure 6) than in the concurrent testing condition [Experiment 1 , Figure $4 ; F(1,8)=7.07, p<.05]$, whereas no such effect was observed in the EC- or sham-lesioned rats.

\section{Discussion}

The present experiment demonstrated that even though the two presurgically acquired discriminations were

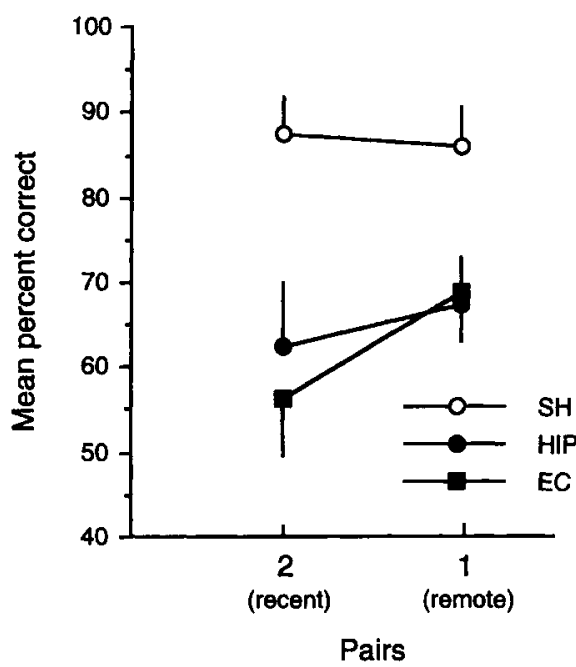

Figure 6. Postoperative retention performance (mean percent correct $\pm S E M$ ) based on the first 16 trials for the two one-pair discriminations in the sham-lesioned (SH), hippocampal-lesioned (HIP), and entorhinal-cortex-lesioned (EC) rats. During retention memory testing, the two pairs were presented one at a daily session (successive memory testing, Experiment 2).

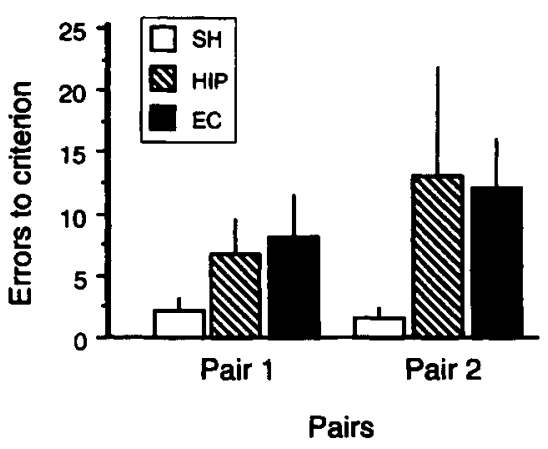

Figure 7. Mean errors $( \pm S E M)$ to reattain criterion performance during relearning of the two pairs postoperatively in the shamlesioned (SH), hippocampal-lesioned (HIP), and entorhinalcortex-lesioned (EC) rats.

tested via a testing procedure identical to that used for initial preoperative training, both the HIP and EC lesions still produced a retention deficit. This result suggests that the retention deficit is due to a retrograde amnesic effect. The results also indicate that the change of the experimental procedure was not a main cause of the retention deficit in the HIP- and EC-lesioned rats used in Experiment 1. However, the HIP-lesioned rats showed significant impairment in learning a new two-pair concurrent discrimination, supporting the idea that the observed retention deficits in Experiment 1 could have been due to an anterograde amnesic effect. These anterograde and retrograde amnesic effects of the lesions, together with results obtained in Experiment 1, are consistent with those reported not only in rats (Eichenbaum et al., 1992; Fidelman, Cho, \& Kesner, personal communication, 1993; O'Keefe \& Nadel, 1978; Olton \& Shapiro, 1992; Wible, Shiber, \& Olton, 1993), but also in nonhuman primates (Moss, Mahut, \& Zola-Morgan, 1981; Zola-Morgan, Squire, Amaral, \& Suzuki, 1989; Zola-Morgan, Squire, Clower, \& Rempel, 1993) and in humans (Oscar-Berman \& Zola-Morgan, 1980; Squire, Zola-Morgan, \& Chen, 1988).

\section{GENERAL DISCUSSION}

The results of both experiments indicate that the HIP lesions produced a retention deficit that could be interpreted to reflect either a retrograde and/or an anterograde amnesic effect. This interpretation is based on the following observations: (1) Rats with HIP lesions were impaired in retention and reacquisition of spatial discriminations in either successive or concurrent testing procedures, (2) rats with HIP lesions were impaired in learning a new two-pair concurrent spatial discrimination, and (3) there was no evidence of a temporal gradient. Even though the absence of a temporal gradient might have been due to a short (3-5 day) learning to surgery interval, especially for the first pair, the time interval was sufficient to uncover a temporal gradient for the EC-lesioned group. The observation that rats with 


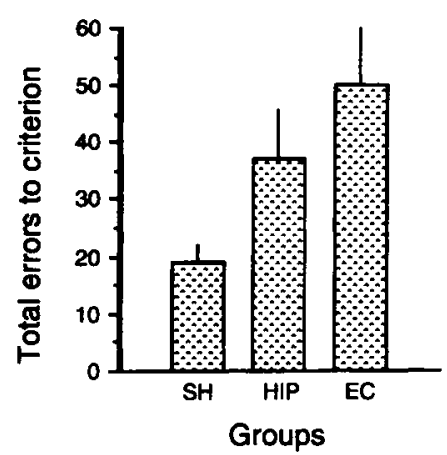

Figure 8. Mean errors ( $\pm S E M$ ) required to learn a new two-pair concurrent discrimination that the animals had not preoperatively learned in the sham-lesioned (SH), hippocampal-lesioned (HIP), and entorhinal-cortex-lesioned (EC) rats.

HIP lesions could learn normally a single spatial discrimination suggests the possibility that the deficit in Experiment 2 might also reflect some effect on retrograde amnesia.

In contrast, the present data indicate that lesions of the EC produce a retention deficit that might be interpreted to reflect a retrograde amnesic effect. This is based on the observation that there was a retention but not a reacquisition deficit in Experiment 1 and that there was a temporal gradient of RA in Experiment 2. These data are in agreement with those obtained with mice with ibotenate lesions of the EC (Cho et al., 1993), indicating that the EC is important for remembering spatial two-choice discrimination for a limited time. The impairment in learning the new two-pair concurrent discrimination probably reflects an enhanced susceptibility to (temporal) interference that is associated with the concurrent discrimination testing procedure.

The PC lesions, however, did not impair retention of the spatial discrimination memory. The $\mathrm{PC}$ has been reported to play a prominent role in the formation of an internal representation of novel spatial environments, and animals with damage to the $\mathrm{PC}$ are unable to utilize spatial cues and to extract the relevant cue from a larger set of irrelevant cues in a specific environment (Benton, 1969; DeRenzi, 1982; DiMattia \& Kesner, 1988a; Kolb \& Walkey, 1987). However, significant retention deficits have been observed following PC lesions in Morris water maze tasks (DiMattia \& Kesner, 1988b) and in cheese board tasks (Kesner et al., 1989), but not in radial maze tasks. This seems to support the idea that there might be an eventual difference in spatial memory load between the two maze tasks, probably because the radial maze offers local views that would help an animal in discriminating among different locations. It should be noted, however, that in a subsequent study, PC lesions produced an overall deficit in retention of four presurgically acquired successive spatial discriminations (Cho \& Kesner, in press) instead of the two discriminations used in the present study. These data from the PC-lesioned rats seem to suggest that the degree of complexity or the amount of information to be processed might determine the extent of the retention deficit following the PC lesions. Consistent with this idea, rats with $\mathrm{PC}$ lesions were reported to be impaired on complex but not simple spatial pattern discrimination tasks (Boyd \& Thomas, 1977) and humans with the PC damage typically were reported to be unable to integrate simple spatial stimuli into a composite whole (Paterson \& Zangwill, 1944).

It has been assumed that the HIP is important for the expression of flexible memory representations, but not for retrieval of the original learning event (Eichenbaum et al., 1994). The present results are partially consistent with this idea since the use of successive pair learning resulted in improved retention scores as compared to concurrent learning, but did not totally prevent the deficits observed in the HIP-lesioned rats. Since the memory representations were formed with the intact HIP, the present data might also be considered to support the previous theory in the sense that this region is critical for normal retrieval of the previously stored relational (spatial) representations.

One additional hypothesis that motivated the present investigation was how variation of mnemonic demands generated by single versus concurrent discrimination paradigms would influence memory performance in an attempt at better defining the nature of anterograde amnesic effect (Eichenbaum et al., 1992; Olton \& Shapiro, 1992). The HIP lesion or fornix transection has been revealed to impair multiple-pair concurrent discriminations but not single-pair discriminations for nonspatial information in monkeys (Mahut, Moss, \& Zola-Morgan, 1981; Moss et al., 1981) and in rodents (Aggleton, Kentridge, \& Sembi, 1993; Eichenbaum et al., 1992; Olton \& Shapiro, 1992; Rothblat, Vnek, Gleason, \& Kromer, 1993; Wible et al., 1992). Moreover, the involvement of the EC and parahippocampal regions in concurrent but not in single discrimination has also been reported in these species (Gaffan \& Murray, 1992; Jarrard, 1993; Moss et al., 1981; Otto, Schottler, Staubli, Eichenbaum, \& Lynch, 1991; Rothblat et al., 1993; Zola-Morgan et al., 1993). The two types of tasks differed in the number of pair-stimuli presented during a test session, and thus in the mnemonic charge associated with a variable degree of interference (Olton \& Shapiro, 1992) or necessity of relational memory processing (Eichenbaum et al., 1992, 1994).

Comparison between performance observed in relearning and new learning of the single-pair discrimination might provide insight into an eventual difference in the utilization of cognitive processes. The observation of the transient retention deficit in the initial phase of postoperative training together with a more or less normal relearning and new learning capability in the HIP- and EClesioned rats is in agreement with data obtained in monkeys (Gaffan \& Lim, 1991; Gaffan \& Murray, 1992; Malamut, Saunder, \& Mishkin, 1984). Since retention testing requires the animals to change their behavior in some way, it could be that the relearning processes are due to time-dependent processes involved in the organi- 
zation of processes necessary for eliminating irrelevant behavior in addition to those involved in temporary storage/retrieval of information. Such procedural subcomponents of a task might partially contribute to retention performance, and a new discrimination might be learned without the HIP and medial temporal regions using alternate cognitive processes. Along the same line of reasoning, Fenton and Bures (1993) have reported that place representations established using only a unilateral functional HIP became unavailable for retrieval by the contralateral functional HIP in overtrained rats, but not in animals that had received only limited training on a Morris water maze task.

The present research investigated further RA for spatial discrimination associated with the parametric manipulation in number of discriminations acquired prior to surgery on the one hand, and in time interval between discriminations as well as in the learning-surgery interval on the other hand, using rats as subjects. The major findings of the present study suggest that the previous factors might interact in a complex manner to influence postoperative retention performance following HIP, EC, and PC lesions.

\section{REFERENCES}

Aggleton, J. P., Kentridge, R. W., \& Sembi, S. (1993). Lesions of the fornix but not the amygdala impair the acquisition of concurrent discriminations by rats. Behavioural Brain Research, 48, 103-112.

Benton, A. L. (1969). Disorders of space orientation. In P. J. Vinken \& G. W. Bruyen (Eds.), Handbook of clinical neurology (pp. 212 228). Amsterdam: Elsevier, North-Holland.

Boyd, M. G., \& Thomas, R. K. (1977). Posterior association cortex lesions in rats: Mazes, pattern discrimination, and reversal learning. Physiological Psychology, 5, 455-461.

BurEš, J., \& BurEŠová, O. (1963). Cortical spreading depression as a memory disturbing factor. Journal of Comparative \& Physiological Psychology, 56, 268-272.

Cho, Y. H., Beracochea, D., \& JafFard, R. (1993). Extended temporal gradient for retrograde and anterograde amnesia produced by ibotenate entorhinal cortex lesions in mice. Journal of Neuroscience, 13, 1759-1766.

Cho, Y. H., \& KeSNer (in press). Involvement of entorhinal cortex or parietal cortex in long-term spatial discrimination memory in rats: Retrograde amnesia. Behavioral Neuroscience.

ChORover, S., \& SCHILleR, P. H. (1965). Short-term retrograde amnesia in rats. Journal of Comparative \& Physiological Psychology, 59, 73-78.

DeREnZI, E. (1982). Disorders of space exploration. New York: Wiley.

DiMatTiA, B. V., \& Kesner, R. P. (1988a). Role of the posterior parietal association cortex in the processing of spatial event information. Behavioral Neuroscience, 102, 397-403.

DiMattia, B. V., \& KeSneR, R. P. (1988b). Spatial cognitive maps: Differential role of parietal cortex and hippocampal formation. Behavioral Neuroscience, 102, 471-480.

Eichenbaum, H., OtTo, T., \& Cohen, N. J. (1992). The hippocampus-What does it do? Behavioral \& Neural Biology, 57, 2-36.

Eichenbaum, H., Otto, T., \& Cohen, N. (1994). Two functional components of the hippocampal memory system. Behavioral \& Brain Sciences, 17, 449-518.

Fenton, A. A., \&. BurEš, J. (1993). Place navigation in rats with unilateral tetrodotoxin inactivation of the dorsal hippocampus: Place but not procedural learning can be lateralized to one hippocampus. Behavioral Neuroscience, 107, 552-564.

Flexner, J. B., Flexner, L. B., \& Stellar, E. (1963, July 5). Memory in mice is affected by intracerebral puromycin. Science, 141, 57-59.
GAFFAN, D. (1993). Normal forgetting impaired acquisition in memory for complex naturalistic scenes by fornix-transected monkeys. Neuropsychologia, 31, 403-406.

GAFFAN, D., \& LiM, C. (1991). Hippocampus and the blood supply to TE: Parahippocampal pial section impairs visual discrimination learning in monkeys. Experimental Brain Research, 87, 227-231.

GafFan, D., \& Murray, E. A. (1992). Monkeys (Macaca facicularis) with rhinal cortex ablations succeed in object discrimination learning despite 24-hr intertrial intervals and fail at matching to sample despite double sample presentations. Behavioral Neuroscience, 106, 30-38.

GolD, P. E., \&. STernberg, D. B. (1978, July 28). Retrograde amnesia produced by several treatments: Evidence for a common neurobiological mechanism. Science, 201, 367-369.

JARRARD, L. E. (1993). On the role of the hippocampus in learning and memory in the rat. Behavioral \& Neural Biology, 60, 9-26.

Juncker, M., Kametani, H., Bresnahan, E. L., \& Ingram, D. K. (1990). Parietal cortex lesions do not impair retention performance of rats in a 14-unit T-maze unless hippocampal damage is present. Physiology \& Behavior, 47, 207-212.

Kesner, R. P., \& DiMatTia, B. V. (1984). Posterior parietal association cortex and hippocampus: Equivalency of mnemonic function in animals and humans. In L. R. Squire \& N. Butters (Eds.), Neuropsychology of memory (pp. 385-398). New York: Guilford.

KeSNER, R. P., FARNSWORTH, G., \& DiMatTia, B. V. (1989). Double dissociation of egocentric and allocentric space following medial prefrontal and parietal cortex lesions in the rat. Behavioral Neuroscience, 103, 956-961.

KESNER, R. P., FARNSWORTH, G., \& KAMETANi, H. (1991). Role of parietal cortex and hippocampus in representing spatial information. Cerebral Cortex, 1, 367-373.

KIM, J. J., \& FANSELOW, M. S. (1992, May 1). Modality-specific retrograde amnesia of fear. Science, 256, 675-677.

KoLB, B., \& WALKEY, J. (1987). Behavioural and anatomical studies of the posterior parietal cortex in the rat. Behavioural Brain Research, 23, 127-145.

MahuT, M., Moss, M., \& Zola-Morgan, S. (1981). Retention deficits after combined hippocampal resections in the monkey. Neuropsychologia, 19, 201-225.

Malamut, B. L., Saunders, R. C., \& Mishkin, M. (1984). Monkeys with combined amygdalo-hippocampal lesions succeed in object discrimination learning despite 24-hour intertrial intervals. Behavioral Neuroscience, 98, 759-769.

McGaugh, J. L. (1966, September 16). Time-dependent processes in memory storage. Science, 153, 1351-1358.

Moss, M., Mahut, H., \& Zola-Morgan, S. (1981). Concurrent discrimination learning of monkeys after hippocampal, entorhinal, or fornix lesions. Journal of Neuroscience, 1, 227-240.

O'KeEFE, J., \& NADEL, L. (1978). The hippocampus as a cognitive map. Oxford: Oxford University Press.

Olton, D. S., \&. ShapiRo, M. L. (1992). Mnemonic dissociations: The power of parameters. Journal of Cognitive Neuroscience, 4, 200207.

Oscar-Berman, M., \& Zola-Morgan, S. (1980). Comparative neuropsychology and Korsakoff's syndrome: II. Two-choice visual discrimination learning. Neuropsychologia, 18, 513-525.

Otto, T., Schottler, F., Staubli, U., Eichenbaum, H., \& LynCh, G. (1991). Hippocampus and olfactory discrimination learning: Effects of entorhinal cortex lesions on olfactory learning and memory in a successive-cue, go-no-go task. Behavioral Neuroscience, 105, 111 119.

Paterson, A., \& Zangwill, O. L. (1944). Disorders of visual space perception associated with lesions of the right cerebral hemisphere. Brain, 67, 331-358.

Rothblat, L. A., VNex, N., Gleason, T. C., \& Kromer, L. F. (1993). Role of the parahippocampal region in spatial and non-spatial memory: Effects of parahippocampal lesions on reward alternation and concurrent object discrimination learning in the rat. Behavioural Brain Research, 55, 93-100.

Salmon, D. P., Zola-Morgan, S., \& Squire, L. R. (1987). Retrograde amnesia following combined hippocampus-amygdala lesions in monkeys. Psychobiology, 15, 37-47. 
SQUiRE, L. R. (1992). Memory and the hippocampus: A synthesis from findings with rats, monkeys, and humans. Psychological Review, 99. 195-231.

Squire, L. R., Cohen, N. J., \& Nadel, L. (1984). The medial temporal region and memory consolidation: A new hypothesis. In $\mathrm{H}$. Weingartner \& E. S. Parker (Eds.), Memory consolidation: Psychobiology of cognition (pp. 185-210). Hillsdale, NJ: Erlbaum.

Squire, L. R., Zola-Morgan, S., \& Chen, K. S. (1988). Human amnesia and animal models of amnesia: Performance of amnesic patients on tests designed for the monkey. Behavioral Neuroscience, 102, 210-221.

Staubli, U., Fraser, D., Kessler, M., \& Lynch, G. (1986). Studies on retrograde and anterograde amnesia of olfactory memory after denervation of the hippocampus by entorhinal cortex lesions. Behavioral \& Neural Biology, 46, 432-444.

Wible, C. G., Shiber, J. R., \& Olton, D. S. (1992). Hippocampus, fimbria-fornix, amygdala, and memory: Object discriminations in rats. Behavioral Neuroscience, 106, 751-761.
WINOCUR, G. (1990). Anterograde and retrograde amnesia in rats with dorsal hippocampal or dorsomedial thalamic lesions. Behavioural Brain Research, 38, 145-154.

Zola-Morgan, S., \& SQuire, L. R. (1990, October 12). The primate hippocampal formation: Evidence for a time-limited role in memory storage. Science, 250, 288-290.

Zola-Morgan, S., SQuire, L. R., Amaral, D. G., \& Suzuki, W. A. (1989). Lesions of perirhinal and parahippocampal cortex that spare the amygdala and hippocampal formation produce severe memory impairment. Journal of Neuroscience, 9, 4355-4370.

Zola-Morgan, S., Squire, L. R., Clower, R. P., \& Rempel, N. L. (1993). Damage to the perirhinal cortex exacerbates memory impairment following lesions to the hippocampal formation. Journal of Neuroscience, 13, 251-265.

(Manuscript received September 19, 1994; revision accepted for publication May 2, 1995.) 